\title{
Is Adherence to Weight Monitoring or Weight-Based Diuretic Self-Adjustment Associated with Fewer Heart Failure-Related Emergency Department Visits or Hospitalizations?
}

\author{
Christine D. Jones, MD ${ }^{1,2}$, George M. Holmes, PhD $^{3,5}$, Darren A. DeWalt, MD, MPH $^{4,5}$, Brian \\ Erman, MSPharm ${ }^{5}$, Kimberly Broucksou, MSW, MPA ${ }^{5}$, Victoria Hawk, RD, MPH ${ }^{5}$, Crystal \\ Cene, MD, MPH ${ }^{4}$, Jia-Rong Wu, RN, PhD $^{6}$, and Michael Pignone, MD, MPH ${ }^{4,5}$ \\ ${ }^{1}$ Department of Social Medicine, Preventive Medicine Residency, at the University of North \\ Carolina at Chapel Hill \\ ${ }^{2}$ Department of Epidemiology, at the University of North Carolina at Chapel Hill \\ ${ }^{3}$ Department of Health Policy and Management, at the University of North Carolina at Chapel Hill \\ ${ }^{4}$ Division of General Medicine and Clinical Epidemiology, at the University of North Carolina at \\ Chapel Hill \\ ${ }^{5}$ The Cecil G. Sheps Center for Health Services Research, at the University of North Carolina at \\ Chapel Hill \\ ${ }^{6}$ The School of Nursing at the University of North Carolina at Chapel Hill
}

\begin{abstract}
Background-Heart failure (HF) self-care interventions can improve outcomes, but less than optimal adherence may limit their effectiveness. We evaluated if adherence to weight monitoring and diuretic self-adjustment was associated with HF-related ED visits or hospitalizations.
\end{abstract}

Methods and Results-We performed a case-control analysis nested in a HF self-care randomized trial. Participants received HF self-care training including weight monitoring and diuretic self-adjustment, which they were to record in a diary. We defined cases as HF-related ED visits or hospitalizations and the 7 preceding days; controls were defined as 7-day periods free of ED visits and hospitalizations. We used logistic regression to compare weight monitoring and diuretic self-adjustment adherence in cases and controls, adjusted for demographic and clinical covariates. Among 303 participants, we identified $81 \mathrm{HF}$-related ED visits or hospitalizations (cases) in 54 patients over one year of follow-up. Weight monitoring adherence (OR $0.42,95 \%$ CI $0.23,0.76$ ) and diuretic self-adjustment adherence (OR $0.44,95 \%$ CI $0.19,0.98$ ) were both associated with lower adjusted odds of HF-related ED visits or hospitalizations.

Conclusions-Adherence to weight monitoring and diuretic self-adjustment was associated with lower odds of HF-related ED visits or hospitalizations. Adherence to these activities may reduce HF-related morbidity.

(C) 2012 Elsevier Inc. All rights reserved

Address for correspondence: Christine DeLong Jones, MD UNC Preventive Medicine Residency Program 121B MacNider Building, CB\# 7240; Chapel Hill, NC 27599-7240 Phone: 919-843-8267, Fax: 919-843-1201, cdjones@ unch.unc.edu.

Publisher's Disclaimer: This is a PDF file of an unedited manuscript that has been accepted for publication. As a service to our customers we are providing this early version of the manuscript. The manuscript will undergo copyediting, typesetting, and review of the resulting proof before it is published in its final citable form. Please note that during the production process errors may be discovered which could affect the content, and all legal disclaimers that apply to the journal pertain.

Disclosures The authors have no conflicts of interest to disclose. 


\section{Keywords}

Congestive Heart Failure; Compliance

\section{Introduction}

Weight monitoring and weight-based diuretic adjustment are frequently recommended as part of heart failure (HF) self-care to reduce volume overload and prevent HF exacerbations, though the evidence to support these recommendations is not robust. ${ }^{1,2}$ Even though $\mathrm{HF}$ self-care programs have been associated with reduced hospitalizations in prior metaanalyses, ${ }^{3,4}$ not all HF self-care clinical trials have demonstrated improved outcomes. ${ }^{3-6}$ Components vary among HF self-care programs, which may partially explain differences in outcomes between trials. In addition, varying adherence to HF self-care programs may explain differences in outcomes. Little is known about the effect of adherence to HF selfcare activities such as weight monitoring and diuretic self-adjustment on outcomes including HF-related ED visits or hospitalizations.

To explore whether adherence to (1) weight monitoring or (2) weight-based diuretic selfadjustment was related to the outcomes of HF-related ED visits or hospitalizations, we conducted a nested case-control study within the intensive intervention arm of a randomized clinical trial of HF self-care training. ${ }^{7}$ We hypothesized that optimal adherence with weight monitoring and diuretic self-adjustment would be associated with lower odds of HF-related ED visits or hospitalizations compared with less than optimal adherence.

\section{Methods}

We conducted a nested case-control study among 303 patients with HF who were assigned to an intensive self-care intervention within a randomized controlled trial comparing different levels of self-care training (ClinicalTrials.gov NCT0037950). Details of this trial, including recruitment procedures and full inclusion and exclusion criteria, have been described previously and will be summarized here. ${ }^{7}$ Briefly, patients participating in this study were diagnosed with either systolic heart failure or heart failure with preserved ejection fraction with New York Heart Association (NYHA) class II-IV symptoms within the prior 6 months, were on a loop diuretic, had adequate cognitive function, and were fluent in either English or Spanish. Patients were recruited between 2006 and 2009 from Internal Medicine and Cardiology clinics at four academic institutions: University of North Carolina, Northwestern University, Olive View - UCLA Medical Center, and University of California, San Francisco - San Francisco General Hospital. Over the course of one year, outcomes were collected, including death; all-cause and HF-related hospitalization; and allcause and HF-related ED visits. The Institutional Review Boards from each site approved the protocol and all patients completed informed consent.

\section{Description of the Intervention}

Over the course of one year, intervention participants received an in-person 40 minute education session followed by a median of 15 educator calls that reinforced weight monitoring, taking proper diuretic doses, medication adherence, salt avoidance, and exercise. Among the four educators who delivered the intervention, two were registered dieticians with experience counseling patients in clinical settings; the other two had bachelor's degrees and previous experience working as health educators. The educators convened for a one day training prior to enrollment and participated in weekly calls with an investigator to develop the educational protocol and ensure similar education delivery across 
sites. All participants were provided a digital bathroom scale and a specialized diary in which they were to record their daily weight and diuretic dose. Participants who were identified by their educator and provider as able to weigh daily and safely perform diuretic self-adjustment were trained individually to perform weight-based diuretic self-adjustment (72\% of 303 intervention arm participants) as directed from a standardized algorithm, termed the "Water Pill Guide" (Figure 1). Patients who were unable to weigh themselves or whose providers felt that they could not safely perform this activity were not taught diuretic self-adjustment. At enrollment the patient's provider identified the patient's euvolemic, or "green zone" weight in this algorithm, for which patients were instructed to take their usual diuretic dose. Patients were also taught to adjust their diuretic dose up or down if their weight went into a "yellow zone," defined by \pm 4-7 pounds from their euvolemic weight. If the patient's weight deviated from the target weight by 8 pounds or more into the "red zone," they were instructed to call their provider immediately in addition to taking the "yellow zone" diuretic dose.

Participants were instructed to mail their weight and diuretic dose diaries back to the educator on a monthly basis in self-addressed, stamped envelopes. The diaries were reviewed by the educator to assess how well the participant was following the protocol and to adjust the target/goal weight with the patient's provider as needed. Diaries were entered into a database by trained study personnel at the conclusion of the study.

\section{Data Collection}

A trained research assistant (RA) at each site administered baseline questionnaires. Socioeconomic status was measured with the MacArthur Scale of Subjective Social Status. ${ }^{8}$ Heart failure severity was assessed with structured questions and categorized by New York Heart Association (NYHA) class. Literacy was measured with a Short Test of Functional health Literacy in Adults (s-TOFHLA) and categorized into adequate literacy ( 223 out of 36) or inadequate/marginal literacy $(<23$ out of 36$) .{ }^{9}$ The RA abstracted medical records to collect data about age; gender; prescribed medications including ACE-inhibitors, angiotensin receptor blockers, and beta blockers; and comorbid conditions including atrial fibrillation cardiovascular disease (TIA/stroke or prior MI or angina), chronic kidney disease (eGFR < $60 \mathrm{~mL} / \mathrm{min} / 1.73 \mathrm{~m}^{2}$ ), COPD, diabetes mellitus, hypertension, and systolic dysfunction (ejection fraction $<45 \%$ on echocardiogram).

The University of North Carolina Survey Research Unit collected clinical outcome data from participants through telephone surveys at 1, 6, and 12 months. Participants were asked about any ED visits, hospitalizations or other events that had occurred in the previous time period, and if so, at which hospital(s) the event(s) took place. Upon identification of an ED visit or hospitalization, admission and discharge summaries were requested from the hospital. In addition, any other ED visits or hospitalizations occurring during a 6-month time period at that site were also requested. A search for ED visits or hospitalizations was also performed at the main clinical sites at 12-month follow up, regardless of whether the participant reported outcomes at that site. The records were then reviewed by one of three adjudication committee members masked to intervention status to determine if the hospitalization was heart failure-related. Adjudication committee members used a study protocol and their clinical judgment to determine whether HF was present and whether HF was an important contributing factor to the ED visit or hospitalization, using a 5-point scale. HF-related ED visits leading to hospitalizations during the same visit were categorized as HF-related hospitalizations. The protocol used to define HF-related ED visits and hospitalizations has been described previously in greater detail. ${ }^{7}$ 


\section{Defining Cases and Controls}

The subject of interest in this analysis was the case or control time period. A case time period was comprised of a HF-related ED visit or hospitalization and the 7-days preceding this event. A control time period was comprised of no event (HF-related ED visit or hospitalization) and the 7 days preceding this event-free day. The 7-day time period was chosen based on findings from a prior study in which clinically important weight changes occurred primarily in the 7 days preceding HF-related hospitalizations. ${ }^{10} \mathrm{We}$ allowed all individuals to contribute control time periods, even if they contributed a case time period at another time; we also allowed all individuals to contribute multiple case and control time periods (Figure 2).

In an effort to minimize differences for potentially confounding variables between case and control time periods and to maximize the efficiency of our analysis, we matched 10 controls for each case for age, gender, NYHA class, and study site. The first three matching variables were chosen due to their prior association with HF-related outcomes. ${ }^{10-12}$ Study site was selected as a matching variable due to differences in HF-related ED visits and hospitalizations among the four study sites. The first 7 days following enrollment were excluded from this analysis to allow patients time to establish a pattern of weight monitoring and diuretic self-adjustment prior to assessing adherence.

During the study, 303 participants were enrolled in the intervention arm. Of these, 6 were excluded from this analysis because they were unable to weigh themselves. Among the remaining 297 individuals, we identified 90 case time periods in 54 individuals (case individuals). Case time periods that contained additional ED visits or hospitalizations within the 7 day time period $(n=9)$ were excluded. After exclusions, our analysis included 81 case time periods in 54 case individuals (Figure 2).

After we excluded control time periods occurring in the first 7 days of the trial $(n=1,776)$ and control time periods containing an ED visit or hospitalization $(n=2,000)$, we identified 102,544 potential control time periods in 297 individuals in which the 8th day did not contain any ED visit or hospitalization (either all-cause or HF-related). Of the 102,544 potential control time periods, we identified 68,868 that matched to case time periods. Several of the control time periods matched to multiple case time periods, generating 176,222 possible control pairings with the 81 case time periods. The number of control time periods available as matches for each case time period ranged between 319 and 5,316. Of these matches, 10 control time periods were randomly selected for each case time period. After matching and random selection of control time periods, our analysis included 810 control time periods in 175 individuals (Figure 2). Of the 54 individuals contributing cases (case individuals), 51 contributed at least one control time period as well.

\section{Defining Adherence}

Adherence to daily weight monitoring was determined from the 7 days of weight diary data for each case or control time period and dichotomized into categories of $\geq 6$ and $<6$ out of 7 days to represent optimal and less than optimal weight monitoring adherence, respectively Non-recorded and non-returned weight data were categorized as nonadherent. Adherence to diuretic self-adjustment required (1) being taught and assigned to diuretic self-adjustment, (2) recording a weight, and (3) agreement between the recorded diuretic dose and the weight-dependent dose as recommended by the diuretic self-adjustment plan. Adherence to diuretic self-adjustment was similarly dichotomized into categories of $\geq 6$ and $<6$ out of 7 days with non-recorded diuretic self-adjustment data categorized as nonadherent. The optimal adherence threshold of $\geq 6$ out of 7 days ( $285.7 \%$ ) was chosen as it most closely 
approximates thresholds, ranging from $>75 \%$ to $>95 \%$ adherence, that are used to define optimal medication adherence. ${ }^{13-16}$

\section{Sensitivity Analyses}

To address possible selection bias, we performed several sensitivity analyses. In our initial sensitivity analyses to mitigate selection bias, both case and control periods were drawn from the 54 case individuals. The effect of $\geq 6$ out of 7 days' adherence to weight monitoring and diuretic self-adjustment was evaluated using case time periods and all available control time periods from case individuals (approximately 1 case time period: 322 control time periods), adjusted only for within-case correlation. To address possible selection bias within the diuretic self-adjustment analysis, we completed another analysis only among those participants who were adherent to weight monitoring $\geq 6$ out of 7 days ( 27 case time periods and 270 control time periods). Our final analysis to address selection bias within the diuretic self-adjustment analysis was performed only among those assigned to a diuretic selfadjustment plan $\geq 6$ days during the 7 -day time period (41 case time periods and 410 control time periods). For the latter two sensitivity analyses, matching and exclusion criteria were otherwise identical to the main case-control analysis. Due to the small number of case time periods in the latter two sensitivity analyses, we performed only unadjusted and minimally adjusted analyses with matching variables (age, gender, NYHA class, and study site).

Because the optimal adherence thresholds for weight monitoring and diuretic selfadjustment necessary to impart clinical benefit are unclear, we performed an additional set of sensitivity analyses to examine adherence thresholds other than $\geq 6$ out of 7 days. We performed analyses using a higher 7 out of 7 day (100\%) adherence threshold as well as lower adherence thresholds of 5 out of 7 days $(\geq 71.4 \%)$ and 4 out of 7 days $(\geq 57 \%)$ for weight monitoring and diuretic self-adjustment.

\section{Statistical Analysis}

We compared demographic, clinical and medication data in individuals who contributed case and control time periods for descriptive purposes with a chi-square test for dichotomous variables and Student's t-test for continuous variables. Continuous variables were evaluated for normal distribution; we performed a Wilcoxon-Mann-Whitney test to compare case and control individuals for continuous variables with a distribution that was not normal. We used similar statistical methods to compare characteristics of case and control time periods, which were not corrected for individuals who contributed multiple case and/or control time periods.

We evaluated the effect of weight monitoring adherence preceding HF-related ED visits or hospitalizations in case versus control time periods in both unadjusted and adjusted analyses using conditional logistic regression for matched data. ${ }^{17}$ Diuretic self-adjustment adherence was evaluated in case versus control time periods in the same manner. Minimally adjusted analyses included only the matching variables (age, gender, NYHA class, and study site). The fully adjusted analyses included the matching variables and subjective socioeconomic status, literacy, cardiovascular disease, chronic kidney disease, and systolic dysfunction given their potential to confound the association between adherence to weight monitoring or diuretic self-adjustment and HF-related ED visits or hospitalizations. In addition, we performed a cluster adjustment to account for individuals who contributed multiple case or control time periods. We considered a two-sided $\mathrm{p}$ value of $<0.05$ statistically significant. All data analyses were performed using Stata 12.0 (College Station, TX). 


\section{Results}

Table 1 shows characteristics at the individual level; case "individuals" are those who contributed one or more case time periods at any point during the year of followup and control "individuals" are those who contributed only control time periods during the year of followup. Individuals who contributed both case and control time periods are characterized as cases in this table. The mean age of the overall sample was 60.9 years, nearly half were women (48\%), and just over half had NYHA Class II HF (51\%). Overall, case individuals were similar to control individuals with regard to socio-demographic characteristics and medication use. More case than control individuals had cardiovascular disease (57\% vs. $37 \%$ ), chronic kidney disease (54\% vs. $33 \%$ ), and systolic dysfunction (70\% vs. 53\%). Individuals from the overall sample that contributed neither case nor control time periods $(n=119)$ were older compared to the individuals that contributed case and control time periods (64.5 years versus 60.9 years). In addition, more of the individuals who contributed neither case nor control time periods had NYHA class I HF and fewer had NYHA class II HF compared to individuals contributing case and control time periods (Table 1).

The 81 case time periods included 12 ED visits (15\%) and 69 hospitalizations $(85 \%)$ related to HF. Among the 54 case individuals contributing the 81 case time periods, 38 individuals contributed 1 case time period each, 9 individuals contributed 2 each, and 7 individuals contributed more than 2 case time periods. As shown in Table 2, the case and control time period characteristics were overall comparable with regard to socio-demographic and clinical characteristics with the exception of systolic dysfunction, which was present in $79 \%$ of case versus $61 \%$ of control time periods.

The case and control time periods also differed in adherence ( $\geq 6$ out of 7 days) to weight monitoring (33\% versus $48 \%$, respectively) and adherence to diuretic self-adjustment (10\% versus $22 \%$, respectively). In the overall sample of 891 time periods, $53 \%$ of weight monitoring days (3304 of 6237 days) were categorized as nonadherent for weights, either because a weight diary was not received ( $87 \%$ of nonadherent days) or because weight values were not recorded on diaries that were received (13\% of nonadherent days). In addition, on $72 \%$ of days (4461 of 6237 days) from the overall sample, either weight or diuretic dose diary data were incomplete or not received and categorized as nonadherent for diuretic self-adjustment. Out of the overall sample of 297 individuals, $21 \%$ did not turn in any diary with weight/diuretic doses for the entire study period; the proportion of individuals that did not turn in a diary for the entire study was similar among cases (22\%) and controls $(22 \%)$.

\section{Effect of adherence to weight monitoring}

Adherence to weight monitoring $\geq 6$ out of 7 days yielded lower odds of HF-related ED visits or hospitalizations (Table 3 ). This association was similar in magnitude for models that were unadjusted (odds ratio (OR) $0.49,95 \%$ CI $0.28-0.84$ ), adjusted only for matching variables (OR 0.46; 95\% CI 0.27-0.78), and fully adjusted (OR 0.42, 95\% CI 0.23-0.76).

\section{Effect of adherence to weight-based diuretic self-adjustment}

Diuretic self-adjustment adherence $\geq 6$ out of 7 days was associated with lower odds of HFrelated ED visits or hospitalizations (Table 3). This association was also similar in magnitude in models that were unadjusted (OR $0.43,95 \%$ CI $0.20-0.95$ ), adjusted only for matching variables (OR $0.42,95 \%$ CI $0.19-0.92$ ), and fully adjusted (OR $0.44,95 \%$ CI 0.19-0.98). 


\section{Sensitivity analyses}

Sensitivity analyses of weight monitoring and diuretic self-adjustment adherence of $\geq 6$ out of 7 days within case and control time periods drawn from the 54 case individuals were attenuated but comparable to the results observed in the overall analyses (Table 3 ). In this subanalysis, we found that odds ratios of HF-related ED visits or hospitalizations of 0.63 (95\% CI 0.40-1.02) for weight monitoring adherence and 0.66 (95\% CI 0.34-1.29) for diuretic self-adjustment adherence.

In a subgroup analysis to evaluate adherence to diuretic self-adjustment among cases and controls who were adherent to weight monitoring $\geq 6$ out of 7 days ( 27 cases, 270 controls; Table 3), we found that adherence to diuretic self-adjustment $\geq 6$ out of 7 days yielded odds ratios that were attenuated, but still comparable to the diuretic self-adjustment analysis from the overall sample in unadjusted (OR $0.63,95 \%$ CI $0.27-1.48$ ) and minimally adjusted analyses (OR $0.58,95 \%$ CI $0.23-1.45)$.

Within a subgroup analysis among case and control time periods who were assigned to diuretic self-adjustment for $\geq 6$ days of the 7 day time period (41 cases, 410 controls; Table 3 ), we found that adherence to diuretic self-adjustment $\geq 6$ out of 7 days had a comparable but attenuated effect compared to the diuretic self-adjustment adherence analysis in the overall sample, yielding lower odds of HF-related ED visits or hospitalizations in unadjusted (OR 0.47, 95\% CI 0.20-1.11) and minimally adjusted analyses (OR 0.39, 95\% CI 0.170.92). This suggests that selection bias did not contribute more than a modest amount to the effect observed in the main analysis.

We performed sensitivity analyses of other weight monitoring adherence thresholds and found similar odd ratios to the $\geq 6$ out of 7 day threshold (Table 4). A 7 out of 7 day weight monitoring adherence threshold (compared to $<7 / 7$ days) yielded an odds ratio of 0.35 (95\% CI $0.19-0.67$ for HF-related ED visits or hospitalizations. Thresholds of $\geq 5$ and $\geq 4$ out of 7 days yielded odds ratios of 0.42 (95\% CI $0.23-0.78$ ) and 0.47 (95\% CI $0.27-0.82$ ), respectively. We performed a similar sensitivity analysis for diuretic self-adjustment and found that odds ratios for adherence thresholds of $7, \geq 5$, and $\geq 4$ out of 7 days were attenuated but comparable to the $\geq 6$ out of 7 day threshold, yielding odds ratios of 0.49 (95\% CI 0.21-1.13), 0.60 (95\% CI 0.30-1.18), and 0.54 (0.27-1.11), respectively, for HFrelated ED visits or hospitalizations (Table 4).

\section{Discussion}

In a case-control study, we found that optimal adherence to weight monitoring and diuretic self-adjustment were both associated with lower odds of a HF-related ED visit or hospitalization compared with less than optimal adherence. This relationship was robust to adjustment for possible confounders. In addition, the beneficial effect of adherence to weight monitoring and diuretic self-adjustment persisted in subanalyses designed to minimize selection bias. Our results suggest that optimizing adherence to weight monitoring and diuretic self-adjustment could result in improved outcomes for patients with HF.

Prior meta-analyses have found that HF self-care programs reduce outcomes of hospitalizations and mortality. ${ }^{3,4}$ In a recent Cochrane review that evaluated either telemonitoring or structured telephone support for patients with HF, several studies involved a component of weight monitoring in which participants were to weigh themselves at a certain frequency and provide results to a research team. ${ }^{4}$ Within this review, we identified ten studies ${ }^{18-26}$ that had a component of weight monitoring, of which five studies ${ }^{20,21,23-25}$ reported weight monitoring adherence. The five studies defined weight monitoring adherence variably: two studies defined adherence as the proportion of weight or blood 
pressure transmissions received daily, ${ }^{21,24}$ one study defined adherence as the proportion of telephone calls and weights completed at least every other day, ${ }^{20}$ and the final two defined adherence as the proportion of weights received either daily ${ }^{25}$ or at least every other day. ${ }^{23}$ All five studies reported adherence levels that were $\geq 80 \%$ to their various measures; three of the five studies also reported improvements in the intervention group compared to the control group for outcomes including mortality, hospitalizations, and ED visits. ${ }^{20,21,23}$ In contrast, $55.1 \%$ of patients were calling to report weights and symptoms at least three times a week after 26 weeks of a weight telemonitoring study that did not find reductions in hospitalizations or mortality. ${ }^{5}$ Although optimal adherence to weight monitoring has not consistently been associated with improved outcomes, ${ }^{6,24,25}$ it is possible that less than optimal adherence to HF self-care programs including weight monitoring may contribute to differences in outcomes.

Although medication adherence in patients with HF has been associated with fewer all-cause and HF-related hospitalizations as well as lower healthcare costs, ${ }^{27,28}$ whether adherence to other self-care behaviors including weight monitoring and diuretic self-adjustment improves outcomes is unclear. While important work in this area has been completed using selfreported indices of self-management, ${ }^{29,30}$ few rigorous evaluations of adherence to daily weight monitoring or diuretic self-adjustment have been completed. Evidence for weight monitoring and diuretic self-adjustment adherence relies heavily on subgroup analyses from larger studies. In the Auckland Heart Failure Management Study (AHFMS), a randomized trial of 197 patients with HF to evaluate the effect of weight diaries, attendance in a HF clinic, and HF education over 12 months, a subgroup analysis showed that any use of a diary to record medication adherence, symptoms, and/or weights by participants $(n=76)$ compared to no use of a diary $(\mathrm{n}=24)$ was related to lower mortality ( $11 \%$ of diary users versus $46 \%$ of non-users, $\mathrm{p}<0.0001)$ and more days alive and out of the hospital (334.7 days in diary users versus 217.4 days in non-users, $\mathrm{p}<0.0001$ ). ${ }^{31}$ In addition, participants who weighed themselves at least once a week $(n=51)$ compared to those who weighed themselves less frequently $(\mathrm{n}=25)$ also had fewer all-cause hospital admissions $(71 \%$ versus $44 \%)$ and more total days alive and not hospitalized (344 versus 210 days). ${ }^{31}$

Findings from our analysis in addition to those from the AHFMS suggest that improved adherence to HF self-management programs including weight monitoring and diuretic selfadjustment can improve outcomes. While multiple strategies may be effective in encouraging HF self-care and improving outcomes, suboptimal adherence to even the most beneficial strategy is likely to diminish related outcome improvements. We theorize that HF self-care interventions are more likely to be effective if they include not only an effective self-care program but also a strong component that promotes adherence to this program.

The ideal threshold to define adherence to self-care behaviors including weight monitoring and diuretic self-adjustment is uncertain. The threshold used in the AHFMS analysis to define "regular" weight monitoring was $\geq 1$ out of 7 days. Within the Cochrane review of telemonitoring for HF, a wide range of thresholds was used to define adherence to weights, from once weekly to twice-daily weight monitoring. ${ }^{4,18,21,23-25}$ In prior research, a medication adherence threshold of $\geq 88 \%$ has been associated with fewer events including HF-related ED visits, cardiac rehospitalizations, and mortality. ${ }^{15}$ We explored higher and lower adherence thresholds to both weight monitoring and diuretic self-adjustment to evaluate if odds of HF-related ED visits or hospitalizations would change when compared to our original $\geq 6$ out of 7 day threshold. We found that different weight monitoring adherence thresholds including 7, $\geq 5$, and $\geq 4$ out of 7 days yielded similar odds ratios for HF-related ED visits or hospitalizations. A similar analysis for diuretic self-adjustment adherence thresholds showed attenuated but similar odds ratios for HF-related ED visits or hospitalizations across different levels of adherence. 
Observational studies are potentially subject to selection bias. The "healthy adherer effect" has been used to describe the observation that optimal adherence not only to medications, but also to placebo, has been associated with improved health outcomes, including mortality. ${ }^{32,33}$ To address possible bias from the healthy adherer effect, we performed two subanalyses: one within case individuals only and another within case and control time periods with weight monitoring adherence of $\geq 6$ out of 7 days. In both of these analyses, we found attenuated but comparable odds ratios to our main analysis, which suggests that the healthy adherer effect did not account for more than a small amount of the observed effect. We addressed another form of selection bias within our diuretic self-adjustment analysis by evaluating adherence to diuretic self-adjustment only among those assigned to a diuretic self-adjustment plan for $\geq 6$ out of 7 days. In this analysis, our findings were similar to our main analysis of diuretic self-adjustment adherence, thus suggesting that this source of selection bias did not substantially affect findings in our main analysis.

Our adherence exposure measurement could also have been a source of bias. We may have under-estimated adherence because non-reported data was considered nonadherent, which may have affected our results. However, failure to systematically record and mail in weights and diuretic doses reflects deviation from the recommended self-care program that appears meaningful. Finally, despite baseline differences in the prevalence of systolic dysfunction, cardiovascular disease, and chronic kidney disease between case and control individuals, we did not find that it affected our final results as all variables were accounted for in our fully adjusted model.

With regard to the generalizability of our findings, our sample population had less advanced HF by NYHA class and was younger compared to recent HF self-care studies. ${ }^{4,6}$ In addition, it is unclear whether adherence to weight monitoring and diuretic self-adjustment would be similarly beneficial outside of a clinical trial setting. Strengths of our study include a sample that was drawn from multiple sites, availability of detailed socio-demographic data, adjudicated outcome measurements for HF-related ED visits and hospitalizations, and the novel assessment of diary-based weight monitoring and diuretic self-adjustment adherence with sub-analyses to mitigate possible sources of bias

In conclusion, we found that optimal adherence to daily weight monitoring and weightbased diuretic self-adjustment was associated with lower odds of HF-related ED visits or hospitalizations. While our findings should be interpreted in the context of a case-control study design, we conclude that optimizing adherence to weight monitoring and diuretic selfadjustment as part of HF self-care may contribute to improved outcomes of HF-related ED visits and hospitalizations. Further evaluation of adherence to weight monitoring and diuretic self-adjustment as part of HF self-care may provide valuable insights about the utility of interventions that teach these activities. Furthermore, enhancing adoption and adherence for these self-care behaviors may be an important step in reducing HF-related morbidity.

\section{Acknowledgments}

The authors would like to thank all of the study participants for their valuable contributions.

Funding Sources This study was funded by National Heart, Lung, and Blood Institute grant R01 HL081257 and NIH American Recovery and Reinvestment Act grant A10-0586-001.

\section{References}

1. Hunt SA, Abraham WT, Chin MH, et al. ACC/AHA. Guideline Update for the Diagnosis and Management of Chronic Heart Failure in the Adult: a report of the American College of Cardiology/ 
American Heart Association Task Force on Practice Guidelines (Writing Committee to Update the 2001 Guidelines for the Evaluation and Management of Heart Failure): developed in collaboration with the American College of Chest Physicians and the International Society for Heart and Lung Transplantation: endorsed by the Heart Rhythm Society. Circulation. Sep 20; 2005 112(12):e154235. 2005. [PubMed: 16160202]

2. Piano MR, Prasun MA, Stamos T, Groo V. Flexible diuretic titration in chronic heart failure: where is the evidence? J Card Fail. Nov; 2011 17(11):944-954. [PubMed: 22041332]

3. Klersy C, De Silvestri A, Gabutti G, Regoli F, Auricchio A. A meta-analysis of remote monitoring of heart failure patients. J Am Coll Cardiol. Oct 27; 2009 54(18):1683-1694. [PubMed: 19850208]

4. Inglis SC, Clark RA, McAlister FA, et al. Structured telephone support or telemonitoring programmes for patients with chronic heart failure. Cochrane Database Syst Rev. 2010; (8):CD007228. [PubMed: 20687083]

5. Chaudhry SI, Mattera JA, Curtis JP, et al. Telemonitoring in patients with heart failure. N Engl J Med. Dec 9; 2010 363(24):2301-2309. [PubMed: 21080835]

6. Koehler F, Winkler S, Schieber M, et al. Impact of remote telemedical management on mortality and hospitalizations in ambulatory patients with chronic heart failure: the telemedical interventional monitoring in heart failure study. Circulation. May 3; 2011 123(17):1873-1880. [PubMed: 21444883]

7. DeWalt DA, Broucksou KA, Hawk V, et al. Comparison of a one-time educational intervention to a teach-to-goal educational intervention for self-management of heart failure: design of a randomized controlled trial. BMC Health Serv Res. 2009; 9:99. [PubMed: 19519904]

8. Adler NE, Epel ES, Castellazzo G, Ickovics JR. Relationship of subjective and objective social status with psychological and physiological functioning: preliminary data in healthy white women. Health Psychol. Nov; 2000 19(6):586-592. [PubMed: 11129362]

9. Baker DW, Williams MV, Parker RM, Gazmararian JA, Nurss J. Development of a brief test to measure functional health literacy. Patient Educ Couns. Sep; 1999 38(1):33-42. [PubMed: 14528569]

10. Chaudhry SI, Wang Y, Concato J, Gill TM, Krumholz HM. Patterns of weight change preceding hospitalization for heart failure. Circulation. Oct 2; 2007 116(14):1549-1554. [PubMed: 17846286]

11. Pulignano G, Del Sindaco D, Tavazzi L, et al. Clinical features and outcomes of elderly outpatients with heart failure followed up in hospital cardiology units: data from a large nationwide cardiology database (IN-CHF Registry). Am Heart J. Jan; 2002 143(1):45-55. [PubMed: 11773911]

12. Krumholz HM, Parent EM, Tu N, et al. Readmission after hospitalization for congestive heart failure among Medicare beneficiaries. Arch Intern Med. Jan 13; 1997 157(1):99-104. [PubMed: 8996046]

13. Monane M, Bohn RL, Gurwitz JH, Glynn RJ, Levin R, Avorn J. The effects of initial drug choice and comorbidity on antihypertensive therapy compliance: results from a population-based study in the elderly. Am J Hypertens. Jul; 1997 10(7 Pt 1):697-704. [PubMed: 9234822]

14. Rosen MI, Rigsby MO, Salahi JT, Ryan CE, Cramer JA. Electronic monitoring and counseling to improve medication adherence. Behav Res Ther. Apr; 2004 42(4):409-422. [PubMed: 14998735]

15. Wu JR, Moser DK, De Jong MJ, et al. Defining an evidence-based cutpoint for medication adherence in heart failure. Am Heart J. Feb; 2009 157(2):285-291. [PubMed: 19185635]

16. Schedlbauer A, Davies P, Fahey T. Interventions to improve adherence to lipid lowering medication. Cochrane Database Syst Rev. 2010; (3):CD004371. [PubMed: 20238331]

17. Breslow NE, Day NE. Statistical methods in cancer research. Volume I - The analysis of casecontrol studies. IARC Sci Publ. 1980; (32):5-338. [PubMed: 7216345]

18. Antonicelli R, Testarmata P, Spazzafumo L, et al. Impact of telemonitoring at home on the management of elderly patients with congestive heart failure. J Telemed Telecare. 2008; 14(6): 300-305. [PubMed: 18776075]

19. Balk AH, Davidse W, Dommelen P, et al. Tele-guidance of chronic heart failure patients enhances knowledge about the disease. A multi-centre, randomised controlled study. Eur J Heart Fail. Nov; 2008 10(11):1136-1142. [PubMed: 18790668] 
20. Capomolla S, Pinna G, La Rovere MT, et al. Heart failure case disease management program: a pilot study of home telemonitoring versus usual care. Eur Heart J Suppl. Nov; 2004 6(F):F91-F98.

21. Cleland JG, Louis AA, Rigby AS, Janssens U, Balk AH. Noninvasive home telemonitoring for patients with heart failure at high risk of recurrent admission and death: the Trans-European Network-Home-Care Management System (TEN-HMS) study. J Am Coll Cardiol. May 17; 2005 45(10):1654-1664. [PubMed: 15893183]

22. Galbreath AD, Krasuski RA, Smith B, et al. Long-term healthcare and cost outcomes of disease management in a large, randomized, community-based population with heart failure. Circulation. Dec 7; 2004 110(23):3518-3526. [PubMed: 15531765]

23. Goldberg LR, Piette JD, Walsh MN, et al. Randomized trial of a daily electronic home monitoring system in patients with advanced heart failure: the Weight Monitoring in Heart Failure (WHARF) trial. Am Heart J. Oct; 2003 146(4):705-712. [PubMed: 14564327]

24. Mortara A, Pinna GD, Johnson P, et al. Home telemonitoring in heart failure patients: the HHH study (Home or Hospital in Heart Failure). Eur J Heart Fail. Mar; 2009 11(3):312-318. [PubMed: 19228800]

25. Soran OZ, Pina IL, Lamas GA, et al. A randomized clinical trial of the clinical effects of enhanced heart failure monitoring using a computer-based telephonic monitoring system in older minorities and women. J Card Fail. Nov; 2008 14(9):711-717. [PubMed: 18995174]

26. Woodend AK, Sherrard H, Fraser M, Stuewe L, Cheung T, Struthers C. Telehome monitoring in patients with cardiac disease who are at high risk of readmission. Heart Lung. Jan-Feb;2008 37(1): 36-45. [PubMed: 18206525]

27. Esposito D, Bagchi AD, Verdier JM, Bencio DS, Kim MS. Medicaid beneficiaries with congestive heart failure: association of medication adherence with healthcare use and costs. Am J Manag Care. Jul; 2009 15(7):437-445. [PubMed: 19589011]

28. Murray MD, Young J, Hoke S, et al. Pharmacist intervention to improve medication adherence in heart failure: a randomized trial. Ann Intern Med. May 15; 2007 146(10):714-725. [PubMed: 17502632]

29. Riegel B, Lee CS, Dickson VV, Carlson B. An update on the self-care of heart failure index. J Cardiovasc Nurs. Nov-Dec;2009 24(6):485-497. [PubMed: 19786884]

30. Jaarsma T, Arestedt KF, Martensson J, Dracup K, Stromberg A. The European Heart Failure Selfcare Behaviour scale revised into a nine-item scale (EHFScB-9): a reliable and valid international instrument. Eur J Heart Fail. Jan; 2009 11(1):99-105. [PubMed: 19147463]

31. Wright SP, Walsh H, Ingley KM, et al. Uptake of self-management strategies in a heart failure management programme. Eur J Heart Fail. Jun; 2003 5(3):371-380. [PubMed: 12798837]

32. Simpson SH, Eurich DT, Majumdar SR, et al. A meta-analysis of the association between adherence to drug therapy and mortality. BMJ. Jul 1.2006 333(7557):15. [PubMed: 16790458]

33. McDermott MM, Schmitt B, Wallner E. Impact of medication nonadherence on coronary heart disease outcomes. A critical review. Arch Intern Med. Sep 22; 1997 157(17):1921-1929. [PubMed: 9308504] 


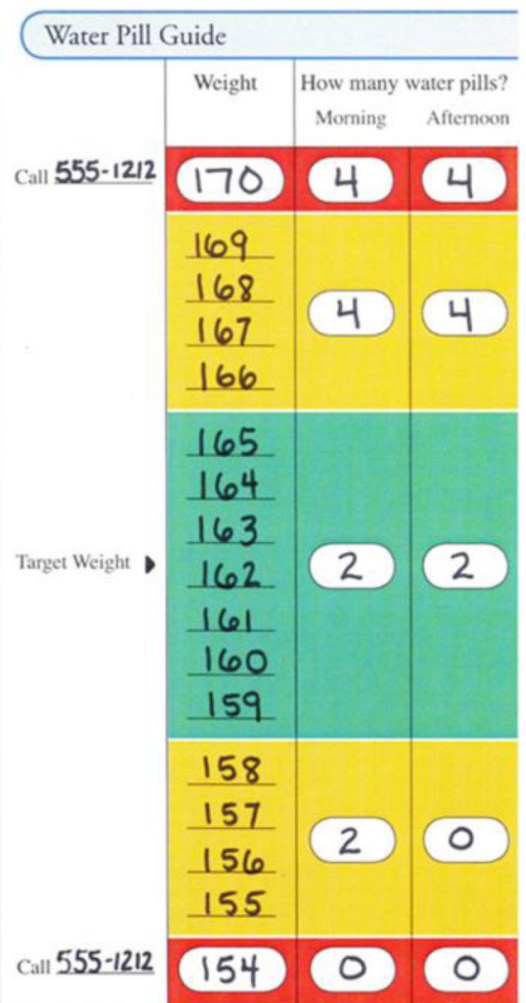

Figure 1.

Example of Water Pill Guide 6 


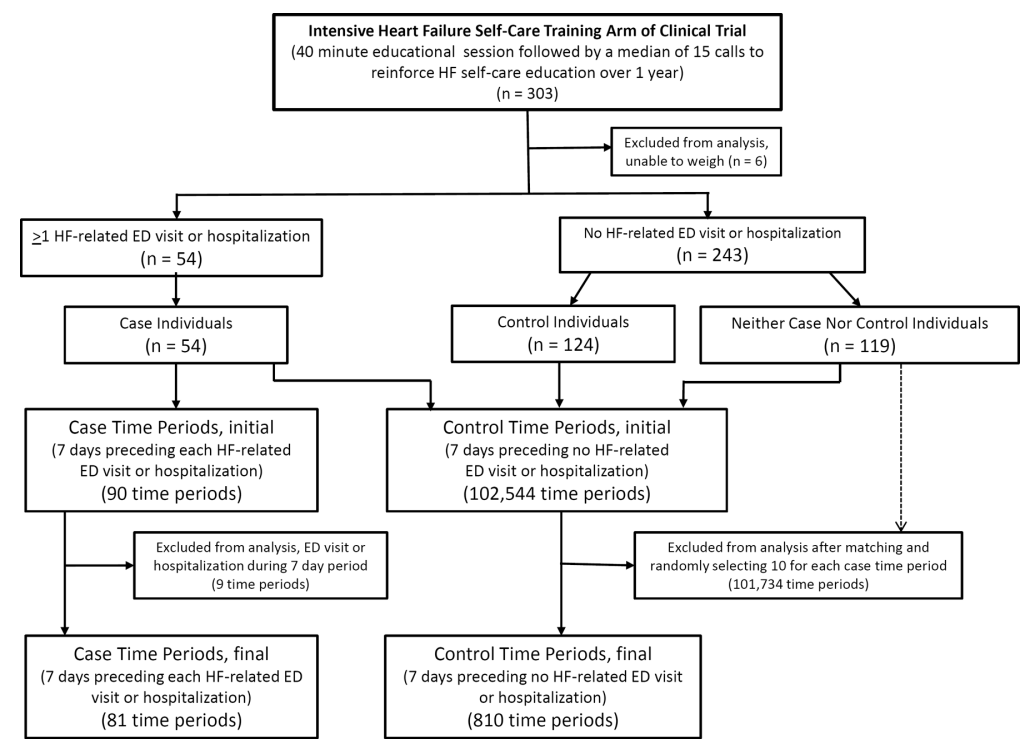

Figure 2.

Flow Chart for Case and Control Time Period Selection 
Table 1

Characteristics of Individuals: Overall Sample, Cases, Controls, and Neither Cases Nor Controls

\begin{tabular}{|c|c|c|c|c|}
\hline Characteristics & $\begin{array}{c}\text { Overall Sample } \\
\text { Individuals }(\mathbf{N}= \\
\text { 297) }\end{array}$ & $\begin{array}{l}\text { Case Individuals } \\
\qquad(\mathbf{N}=\mathbf{5 4})\end{array}$ & $\begin{array}{c}\text { Control } \\
\text { Individuals }(N= \\
124)^{*}\end{array}$ & $\begin{array}{c}\text { Neither Case Nor } \\
\text { Control } \\
\text { Individuals } \\
(\mathbf{N}=119)\end{array}$ \\
\hline Age, mean $(\mathrm{SD})^{\dagger}$ & $60.9(13.8)$ & $59.7(14.2)$ & $58.0(12.9)$ & $64.5(13.9)$ \\
\hline $\operatorname{Men}(\mathrm{N}, \%)$ & $154(52)$ & $29(54)$ & $71(57)$ & $54(45)$ \\
\hline \multicolumn{5}{|l|}{ NYHA Class $(\mathrm{N}, \%)^{\dagger}$} \\
\hline I & $52(18)$ & $7(13)$ & $10(8)$ & $35(29)$ \\
\hline II & $150(51)$ & $29(54)$ & $83(67)$ & $38(32)$ \\
\hline III & $61(21)$ & $12(22)$ & $22(18)$ & $27(23)$ \\
\hline IV & $34(11)$ & $6(11)$ & $9(7)$ & $19(16)$ \\
\hline \multicolumn{5}{|l|}{ Study Site (N, \%) } \\
\hline UNC & $105(35)$ & $22(41)$ & $48(39)$ & $35(29)$ \\
\hline Northwestern University & $81(27)$ & $15(28)$ & $31(25)$ & $35(29)$ \\
\hline UCSF & $74(25)$ & $9(17)$ & $33(27)$ & $32(27)$ \\
\hline Olive View, UCLA & $37(12)$ & $8(15)$ & $12(10)$ & $17(14)$ \\
\hline $\begin{array}{l}\text { Socioeconomic Status, median (interquartile } \\
\text { range) }{ }^{\ddagger}\end{array}$ & $4(3,6)$ & $4(2,5)$ & $4(2,6)$ & $5(3,7)$ \\
\hline Inadequate Literacy $(\mathrm{N}, \%)$ & $109(37)$ & $19(35)$ & $45(36)$ & $45(38)$ \\
\hline Cardiovascular Disease $(\mathrm{N}, \%)^{\xi}$ & $131(44)$ & $31(57)$ & $46(37)$ & $54(45)$ \\
\hline Chronic Kidney Disease $(\mathrm{N}, \%)^{\xi}$ & $128(43)$ & $29(54)$ & $41(33)$ & $58(49)$ \\
\hline Diabetes Mellitus (N, \%) & $148(50)$ & $28(52)$ & $60(48)$ & $60(50)$ \\
\hline Hypertension $(\mathrm{N}, \%)$ & $251(85)$ & $44(81)$ & $104(84)$ & $103(87)$ \\
\hline Systolic Dysfunction $(\mathrm{N}, \%)^{\mathcal{S}}$ & $167(56)$ & $38(70)$ & $66(53)$ & $63(53)$ \\
\hline ACE-Inhibitor or ARB (N, \%) & $235(79)$ & $43(80)$ & $99(80)$ & $93(78)$ \\
\hline Beta-Blocker (N, \%) & $237(80)$ & $45(83)$ & $103(83)$ & $89(75)$ \\
\hline $\begin{array}{l}\text { Taught to perform diuretic self-adjustment }(\mathrm{N} \text {, } \\
\%)\end{array}$ & $219(74)$ & $39(72)$ & $91(73)$ & $89(75)$ \\
\hline
\end{tabular}

Excludes case individuals that contributed control time periods

$t^{\dagger}<0.001$ comparing pooled "case and control individuals" to "neither case nor control individuals"

${ }^{*}$ No significant differences between groups by either t-test or Wilcoxon-Mann-Whitney test

$\xi_{\mathrm{p}}<0.05$ comparing "case individuals" and "control individuals" 
Table 2

Characteristics of Case and Control Time Periods

\begin{tabular}{|c|c|c|c|}
\hline Characteristics & $\begin{array}{l}\text { Combined Case and } \\
\text { Control Time Periods } \\
\qquad(\mathrm{N}=891)\end{array}$ & $\begin{array}{l}\text { Case Time Periods }(\mathrm{N}= \\
\mathbf{8 1})\end{array}$ & $\begin{array}{l}\text { Control Time Periods } \\
\quad(\mathbf{N}=\mathbf{8 1 0})\end{array}$ \\
\hline Age, median (interquartile range) ${ }^{*}$ & $58.9(49.9,68.1)$ & $57.6(51.7,65.5)$ & $58.9(49.9,68.4)$ \\
\hline $\operatorname{Men}(\mathrm{N}, \%)$ & $484(54)$ & $44(54)$ & $440(54)$ \\
\hline \multicolumn{4}{|l|}{ NYHA Class $(\mathrm{N}, \%)$} \\
\hline I & $77(9)$ & $7(9)$ & $70(9)$ \\
\hline II & $495(56)$ & $45(56)$ & $450(56)$ \\
\hline III & $198(22)$ & $18(22)$ & $180(22)$ \\
\hline IV & $121(14)$ & $11(14)$ & $110(14)$ \\
\hline \multicolumn{4}{|l|}{ Study Site (N, \%) } \\
\hline UNC & $385(43)$ & $35(43)$ & $350(43)$ \\
\hline Northwestern University & $264(30)$ & $24(30)$ & $240(30)$ \\
\hline UCSF & $169(19)$ & $13(16)$ & $156(19)$ \\
\hline Olive View, UCLA & $73(8)$ & $9(11)$ & $64(8)$ \\
\hline Socioeconomic Status, median (interquartile range) ${ }^{*}$ & $4(3,7)$ & $4(3,5)$ & $4(3,7)$ \\
\hline Inadequate Literacy $(\mathrm{N}, \%)$ & $295(33)$ & $24(30)$ & $271(33)$ \\
\hline Cardiovascular Disease (N, \%) & $482(54)$ & $45(56)$ & $437(54)$ \\
\hline Chronic Kidney Disease (N, \%) & $353(40)$ & $39(48)$ & $314(39)$ \\
\hline Diabetes Mellitus (N, \%) & $396(44)$ & $39(48)$ & $357(44)$ \\
\hline Hypertension (N, \%) & $714(80)$ & $65(80)$ & $649(80)$ \\
\hline Systolic Dysfunction $(\mathrm{N}, \%)^{\dagger}$ & $559(63)$ & $64(79)$ & $495(61)$ \\
\hline ACE-Inhibitor or ARB (N, \%) & $710(80)$ & $68(84)$ & $642(79)$ \\
\hline Beta-Blocker (N, \%) & $750(84)$ & $69(85)$ & $681(84)$ \\
\hline $\begin{array}{l}\text { Adherent to weight monitoring }{ }^{*} \text { ( } \geq 6 \text { out of } 7 \text { days; } \mathrm{N} \text {, } \\
\% \text { ) }\end{array}$ & $416(47)$ & $27(33)$ & $389(48)$ \\
\hline $\begin{array}{l}\text { Assigned to diuretic self-adjustment }(\geq 6 \text { out of } 7 \text { days; } \\
\mathrm{N}, \%)\end{array}$ & $540(61)$ & $41(51)$ & $499(62)$ \\
\hline $\begin{array}{l}\text { Adherent to diuretic self-adjustment }{ }^{+} \text {( } \geq 6 \text { out of } 7 \text { days; } \\
\mathrm{N}, \% \text { ) }\end{array}$ & $184(21)$ & $8(10)$ & $176(22)$ \\
\hline \multicolumn{4}{|c|}{ * No significant differences between groups by either t-test or Wilcoxon-Mann-Whitney test } \\
\hline \multicolumn{4}{|l|}{$t_{\mathrm{p}=0.002}$} \\
\hline$F_{\mathrm{p}}^{*}<0.05$ & & & \\
\hline
\end{tabular}


Table 3

Association of Adherence to Weight Monitoring and Diuretic Self-Adjustment with HF-Related ED Visits and Hospitalizations; Secondary Analyses in Selected Subgroups

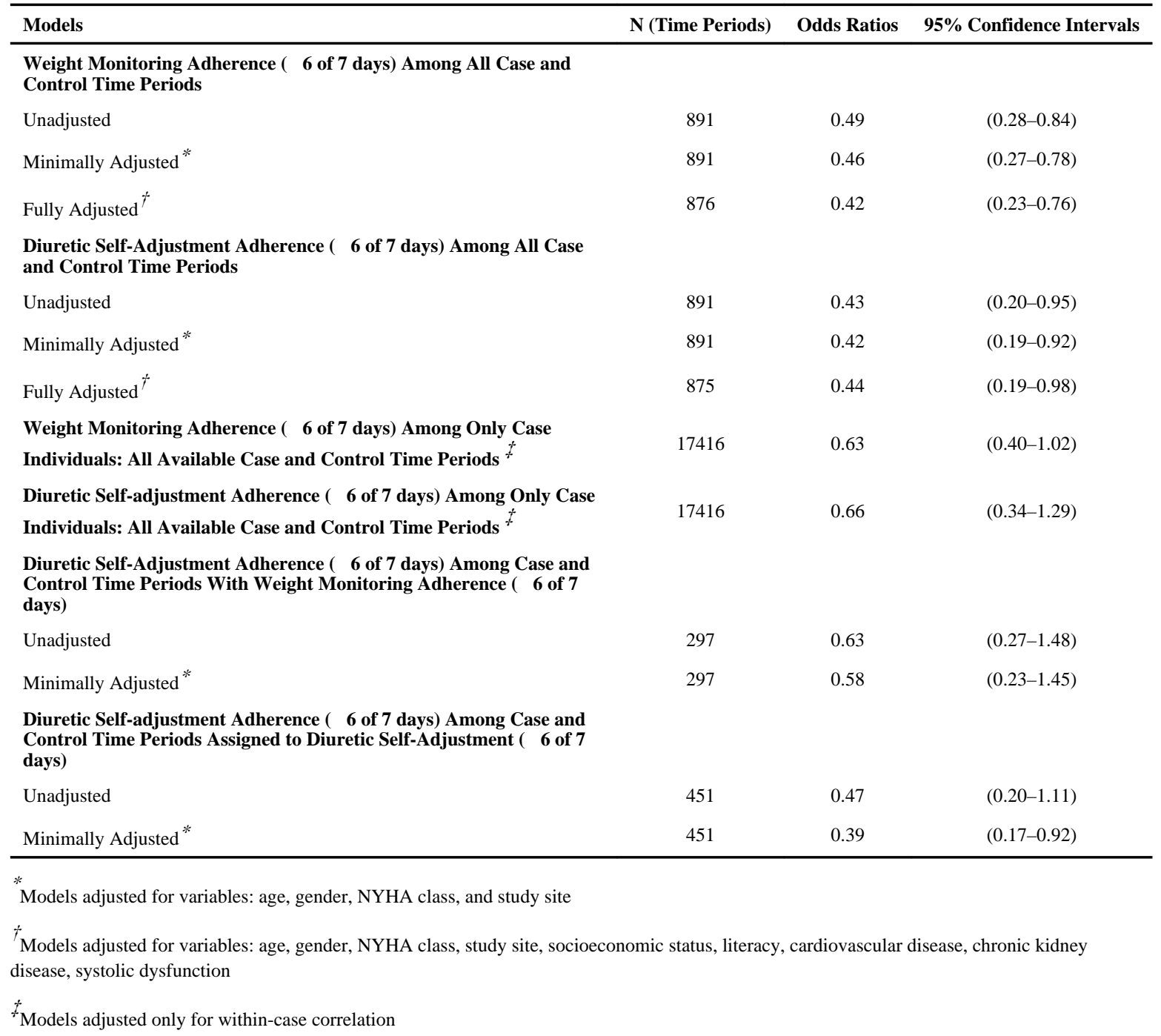


Table 4

Sensitivity Analysis of Variable Adherence Levels to Weight Monitoring and Adherence to Diuretic SelfAdjustment with HF-Related ED Visits and Hospitalizations in Case and control Time Peridos

\begin{tabular}{lccc}
\hline Models & N (Time Periods) & Odds Ratios & 95\% Confidence Intervals \\
\hline $\begin{array}{l}\text { Weight Monitoring Adherence Levels } \\
\quad\end{array}$ & & & \\
7 of 7 days & 876 & 0.35 & $(0.19-0.67)$ \\
$\geq 6$ of 7 days & 876 & 0.42 & $(0.23-0.76)$ \\
$\geq 5$ of 7 days & 876 & 0.42 & $(0.23-0.78)$ \\
$\geq 4$ of 7 days & 876 & 0.47 & $(0.27-0.82)$ \\
Diuretic Self-Adjustment Adherence Levels $*$ & & & \\
7 of 7 days & 875 & 0.49 & $(0.21-1.13)$ \\
$\geq 6$ of 7 days & 875 & 0.44 & $(0.19-0.98)$ \\
$\geq 5$ of 7 days & 875 & 0.60 & $(0.30-1.18)$ \\
$\geq 4$ of 7 days & 875 & 0.54 & $(0.27-1.11)$ \\
\hline
\end{tabular}

* Models adjusted for variables: age, gender, NYHA class, study site, socioeconomic status, literacy, cardiovascular disease, chronic kidney disease, systolic dysfunction 